American Journal of Applied Sciences 7 (4): 540-544, 2010

ISSN 1546-9239

(C) 2010Science Publications

\title{
EEG Signals During Epileptic Seizure as a Semigroup of Upper Triangular Matrices
}

\author{
Faisal Abdurabu Mubarak Binjadhnan and Tahir Ahmad \\ Theoretical and Computational Modeling for Complex Systems, \\ Department of Mathematics, Ibnu Sinu Institute for Fundamental Science Studies, \\ University Technology Malaysia 81310 Skudai, Johor, Malaysia
}

\begin{abstract}
Problem statement: EEG signals during epileptic seizure can be presented as an algebraic structure, namely semigroup of upper triangular matrices. Approach: EEG signals during seizure were recorded and composed into set of matrices. They were transformed into upper triangular matrices using QR-Schur decomposition and finally as a semigroup of upper triangular matrices. Results: EEG signals during epileptic seizure were transformed as a semigroup of upper triangular matrices under matrix multiplication. Conclusion: This study described the procedure to transform signal during epileptic seizure (brainstorm) into an algebraic structure. This is the key step that will enable us to further proceed to obtain some pattern out of the seizure data in our future research.
\end{abstract}

Key words: Electroencephalography, semigroup

\section{INTRODUCTION}

Electroencephalography (EEG) is a recording of electrical activity originating from the brain. It plays an important diagnostic role in epilepsy and provides supporting evidence of a seizure disorder as well as assisting with classification of seizures. EEG has been used extensively to record the abnormal brain activity associated with epileptic seizures. It is recorded on the surface of the scalp using electrodes, thus the signal is retrievable non-invasively. Abnormal patterns such as spikes, sharp waves and, spikes and wave complexes can be recorded. The type of activity and the area of the brain that is recorded from EEG will assist the physician in prescribing the correct medication for certain type of epilepsy. Patients with epilepsy that cannot be controlled by medication will often have surgery in order to remove the damaged tissue. Thus the EEG plays an important role in localizing this tissue.

EEG analysis still relies mostly on its visual inspection. Due to the fact that visual inspection is very subjective and hardly allows any statistical analysis or standardization, several methods were proposed in order to quantify the information of the EEG. Among these, the Fourier Transform emerged as a very powerful tool capable of characterizing the frequency components of EEG signals, even reaching diagnostic importance. However, Fourier Transform has some disadvantages that limit its applicability and therefore, other methods for extracting "hidden" information from the EEG signals was necessary.

Literature review: Fuzzy Topographic Topological Mapping (FTTM) is a novel method for solving neuromagnetic inverse problem to determine the current source, i.e., epileptic foci. FTTM Version 1 has been developed to present a 3-D view of an unbounded single current source (Ahmad et al., 2008b; Yun and Ahmad, 2003) in one angle observation (upper of a head model). Basically, this newly developed model is a topological mapping which contains some fuzzy structures and it comprises four components linked by three different algorithms as shown in Fig. 1.

The four components are Magnetic Contour Plane (MC), Base Magnetic Plane (BM), Fuzzy Magnetic Field (FM) and Topographic Magnetic Field (TM) Fig. 1.

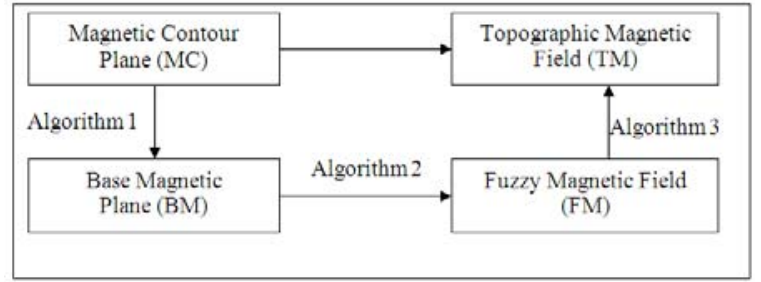

Fig. 1: FTTM version 1

Corresponding Author: Faisal Abdurabu Mubarak Binjadhnan, Theoretical and Computational Modeling for Complex Systems, Department of Mathematics, Ibnu Sinu Institute for Fundamental Science Studies, University Technology Malaysia 81310 Skudai, Johor, Malaysia 
MC is actually a magnetic field on a plane above a current source with $\mathrm{z}=0$. The plane is lowered down to $\mathrm{BM}$, which is a plane of the current source with $\mathrm{z}=-\mathrm{h}$. Then the entire BM is fuzzified into a Fuzzy environment (FM), where all the magnetic field readings are fuzzified. The final process is defuzzification of the fuzzified data to obtain a 3-D view of the current source (TM).

Ahmad et al. (2008a) has developed a new method for mapping high dimensional signal, namely EEG into a low dimensional space (MC). The whole processes of this novel model consisted three main parts. The first part was flattening the EEG where the transformation of three dimensional space into two dimensional space that involved location of sensor in patients head with EEG signal. The second part is the EEG signal was then processed using Fuzzy c-Means. The last part was to find the optimal number of cluster by using cluster validity analysis.

Ahmad's EEG coordinate system Fig. 2a is defined as:

$\mathrm{C}_{\mathrm{EEG}}=\left\{\left((\mathrm{x}, \mathrm{y}, \mathrm{z}), \mathrm{e}_{\mathrm{p}}\right): \mathrm{x}, \mathrm{y}, \mathrm{z}, \mathrm{e}_{\mathrm{p}} \in \mathrm{R}\right.$ and $\left.\mathrm{x}^{2}+\mathrm{y}^{2}+\mathrm{z}^{2}=\mathrm{r}^{2}\right\}$

where, $r$ is the radius of a patient head. She modeled the human's head as a sphere.

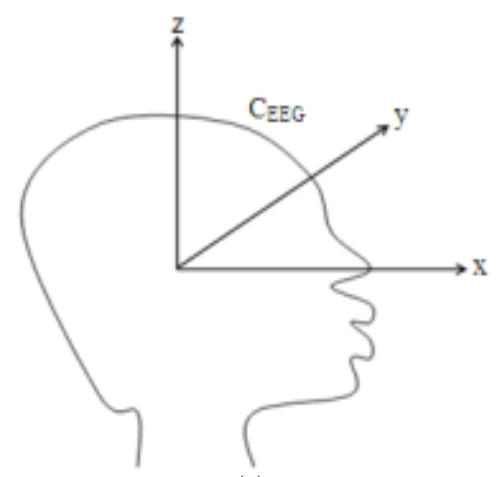

(a)

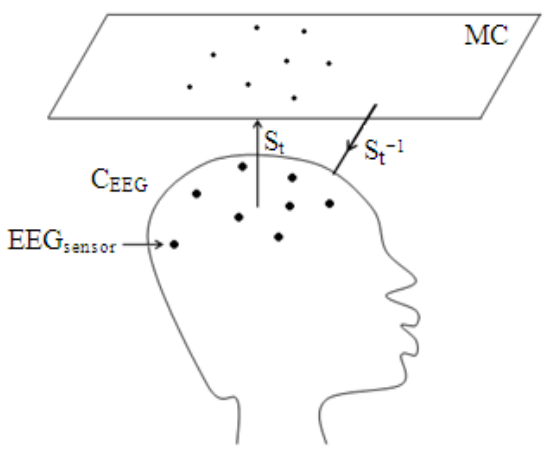

(b)

Fig. 2: (a) EEG coordinate system (b) EEG projection
Furthermore, the mapping of $\mathrm{C}_{\mathrm{EEG}}$ to a plane (MC) is defined as follows.

$\mathrm{S}_{\mathrm{t}}$ : $\mathrm{C}_{\mathrm{EEG}} \rightarrow$ MC Fig. 2b such that:

$S_{t}\left((x, y, z), e_{p}\right)=\left(\frac{r x+i r y}{r+z}, e_{p}\right)=\left(\frac{r x}{r+z}, \frac{r y}{r+z}\right)_{e_{p}(x, y, z)}$

Both $\mathrm{C}_{\mathrm{EEG}}$ and $\mathrm{MC}$ were designed and proven in (Yun and Ahmad, 2003) as 2-manifolds. Ahmad et al. (2008a) also had shown that $S_{t}$ is a one to one function as well as being conformal. With the fact that $S_{t}$ is conformal, therefore the mapping can preserve information, in particular angle and orientation of the surface and EEG signal recorded from the surface of high dimensional into a low dimensional spaces; i.e. mapping EEG signal into a plane. Then, Ahmad et al. (2008a) implements this technique followed by clustering on the real time EEG data obtained from patients who suffer from epileptic seizure.

The signals were digitized at 256 samples per second using Nicolet One EEG software. The average potential difference was calculated from the 256 samples of raw data at every second. Similarly to the position of electrodes, the EEG signal was also preserved during this new method. Subsequently, every single second of the particular average potential difference was stored into a file which contains the position of electrode on MC plane.

\section{MATERIALS AND METHODS}

In our study, we rewrite $\mathrm{MC}$ in terms of square matrices. Therefore every single second of the particular average potential difference was stored into a square matrix which contains the position of electrode on MC plane. Thus Magnetic Contour Plane became a set of $(n \times n)$ square matrices defined as:

$$
\mathrm{MC}_{\mathrm{n}}=\left\{\left[\beta_{\mathrm{ij}}(\mathrm{z})_{\mathrm{t}}\right]_{\mathrm{n} \times \mathrm{n}}: \mathrm{i}, \mathrm{j} \in \mathbf{Z}^{+}, \beta_{\mathrm{ij}}(\mathrm{z})_{\mathrm{t}} \in \mathbf{R}\right\}
$$

where, $\beta_{\mathrm{ij}}(\mathrm{z})_{\mathrm{t}}$ is a potential difference reading of EEG signals from a particular ij sensor at time t.

Semigroup of $\mathbf{M C}_{\mathbf{n}}$ : We are going to show that the nonempty set of square matrices (EEG signals) satisfies all the axioms of a semigroup given in (Whitelaw, 1978) under matrix multiplication. In other words, we are going to show that:

- $\quad \mathrm{MC}_{\mathrm{n}}=\left\{\left[\beta_{\mathrm{ij}}(\mathrm{z})_{\mathrm{t}}\right]_{\mathrm{n} \times \mathrm{n}}: \mathrm{i}, \mathrm{j} \in \mathbf{Z}^{+}, \beta_{\mathrm{ij}}(\mathrm{z})_{\mathrm{t}} \in \mathbf{R}\right\}$ is closed with respect to matrix multiplication

- matrix multiplication on $\mathrm{MC}_{\mathrm{n}}$ is associative 
Theorem 1: The set of $(n \times n)$ square matrices $M C_{n}$ is a semigroup under matrix multiplication.

Proof: Firstly, let us show that $\mathrm{MC}_{\mathrm{n}}$ is closed with respect to matrix multiplication. We pick:

$A=\left(\begin{array}{ccc}\beta 1_{1,1} & \cdots & \beta 1_{1, n} \\ \vdots & \ddots & \vdots \\ \beta 1_{n, 1} & \cdots & \beta 1_{n, n}\end{array}\right)$ and $B=\left(\begin{array}{ccc}\beta 2_{1,1} & \cdots & \beta 2_{1, n} \\ \vdots & \ddots & \vdots \\ \beta 2_{n, 1} & \cdots & \beta 2_{n, n}\end{array}\right) \in \mathrm{MC}_{n}$

Then:

$$
\begin{aligned}
\mathrm{AB} & =\left(\begin{array}{ccc}
\beta 1_{1,1} & \cdots & \beta 1_{1, \mathrm{n}} \\
\vdots & \ddots & \vdots \\
\beta 1_{\mathrm{n}, 1} & \cdots & \beta 1_{\mathrm{n}, \mathrm{n}}
\end{array}\right)\left(\begin{array}{ccc}
\beta 2_{1,1} & \cdots & \beta 2_{1, \mathrm{n}} \\
\vdots & \ddots & \vdots \\
\beta 2_{\mathrm{n}, 1} & \cdots & \beta 2_{\mathrm{n}, \mathrm{n}}
\end{array}\right) \\
& =\left(\sum_{\mathrm{j}=1}^{\mathrm{n}} \beta_{1 \mathrm{i}, \mathrm{j}} \beta 2_{\mathrm{j}, \mathrm{k}}\right)_{(\mathrm{nn})}
\end{aligned}
$$

Notice we go across the i-th row of the first matrix and down the $\mathrm{k}$-th column of the second matrix to obtain the entry in position (i, k):

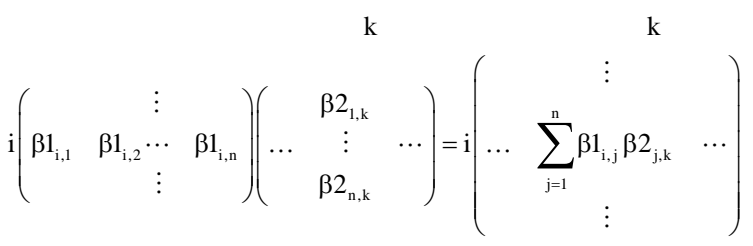

Now $\beta 1_{i, j}, \quad \beta 2_{j, k} \in \square$ for a particular time $t \in \mathbf{R}^{+}$ and without loss of generality, $\beta 1_{i, j} \beta 2_{j, k} \in \mathbf{R}$ for some time $t \in \mathbf{R}^{+}$, thus $\sum_{\mathrm{j}=1}^{\mathrm{n}} \beta 1_{\mathrm{i}, \mathrm{j}} \beta 2_{\mathrm{j}, \mathrm{k}} \in \square$. Since $\mathrm{A}, \mathrm{B} \in \mathrm{MC}_{\mathrm{n}}$ are arbitrary, therefore $A B \in M C_{n}$ and hence $M C_{n}$ is closed with respect to matrix multiplication.

Secondly, let us show that matrix multiplication on $\mathrm{MC}_{\mathrm{n}}$ is associative. Pick:

$$
A=\left[\beta 1_{i, j}\right]_{(n)}, B=\left[\beta 2_{j, k}\right]_{(n n)}, C=\left[\beta 3_{k, l}\right]_{(n n)}
$$

Then:

$$
A B=\left(\sum_{j=1}^{n} \beta 1_{i, j} \beta 2_{j, k}\right)_{(n n)}
$$

$$
\begin{aligned}
(A B) C & =\left(\sum_{k=1}^{n}\left(\sum_{j=1}^{n} \beta 1_{i, j} \beta 2_{j, k}\right) \beta 3_{k, 1}\right)_{(n n)} \\
& =\left(\sum_{k=1}^{n} \sum_{j=1}^{n} \beta 1_{i, j} \beta 2_{j, k} \beta 3_{k, 1}\right)_{(n n)} \\
B C=\left(\sum_{k=1}^{n} \beta 2_{j, k} \beta 3_{k, 1}\right)_{(n n)} & \left(\sum_{j=1}^{n} \beta 1_{i, j}\left(\sum_{k=1}^{n} \beta 2_{j, k} \beta 33_{k, 1}\right)\right)_{(n n)} \\
A(B C) & =\left(\sum_{j=1}^{n} \sum_{k=1}^{n} \beta 1_{i, j} \beta 2_{j, k} \beta 3_{k, 1}\right)_{(n n)}
\end{aligned}
$$

Since:

$\sum_{\mathrm{k}=1}^{\mathrm{n}} \sum_{\mathrm{j}=1}^{\mathrm{n}} \beta 1_{\mathrm{i}, \mathrm{j}} \beta 2_{\mathrm{j}, \mathrm{k}} \beta 3_{\mathrm{k}, \mathrm{l}}=\sum_{\mathrm{j}=1}^{\mathrm{n}} \sum_{\mathrm{k}=1}^{\mathrm{n}} \beta 1_{\mathrm{i}, \mathrm{j}} \beta 2_{\mathrm{j}, \mathrm{k}} \beta 3_{\mathrm{k}, \mathrm{l}}$, we have (AB) $C=A(B C)$. The associativity of $M_{n}$ reveals that historical event is preserved in time (Nehaniv and Dautenhahn, 1998). It means that the property of time is actually embedded in $\mathrm{MC}_{\mathrm{n}}$.

We have shown that:

- $\quad \mathrm{MC}_{\mathrm{n}}=\left\{\left[\beta_{\mathrm{ij}}(\mathrm{z})_{\mathrm{t}}\right]_{\mathrm{n} \times \mathrm{n}}: \mathrm{i}, \mathrm{j} \in \mathbf{Z}^{+}, \beta_{\mathrm{ij}}(\mathrm{z})_{\mathrm{t}} \in \mathbf{R}\right\}$ is closed with respect to matrix multiplication

- matrix multiplication on $\mathrm{MC}_{\mathrm{n}}$ is associative

In other words, Magnetic Contour plane (MC) is a semigroup of square matrices under matrix multiplication.

Triangularization of $\mathbf{M} \mathbf{C}_{\mathbf{n}}$ : There are many methods to triangularize a matrix $\left[\beta_{\mathrm{ij}}(\mathrm{z})_{\mathrm{t}}\right]_{\mathrm{n} \times \mathrm{n}}$ in $\mathrm{MC}_{\mathrm{n}}$. For example, Elementary, Householder and Givens matrices can be used to achieve important matrix factorization such as LU and its variants, QR and reduction to a Hessenberg form of a matrix.

Since our main purpose in this study is to prove $\mathrm{MC}_{n}$ as a semigroup of $(\mathrm{n} \times \mathrm{n})$ upper triangular matrices $\mathrm{MC}_{\mathrm{n}}$ " and every element in $\mathrm{MC}_{\mathrm{n}}$ " as a block matrix then we will choose the Schur decomposition to decompose our square matrix $\left[\beta_{\mathrm{ij}}(\mathrm{z})_{\mathrm{t}}\right]_{\mathrm{n} \times \mathrm{n}}$ in $\mathrm{MC}_{\mathrm{n}}$. 
If the matrix $\mathrm{A}$ is real as in our case, then the real Schur Decomposition is computed, in which the matrix $\mathrm{Q}$ is orthogonal and $\mathrm{U}$ is block upper triangular with blocks of size at most $2 \times 2$ along the diagonal:

$$
Q^{\mathrm{T}} \mathrm{AQ}=\mathrm{U}
$$

Theorem 2 (Datta, 1995): Let A be an $n \times n$ real matrix. Then there exists an $n \times n$ orthogonal matrix $Q$ such that:

$$
\mathrm{Q}^{\mathrm{T}} \mathrm{AQ}=\mathrm{R}=\left(\begin{array}{cccc}
\mathrm{R}_{11} \mathrm{R}_{12} & \cdots & \mathrm{R}_{1 \mathrm{k}} \\
0 & \mathrm{R}_{22} & \cdots & \mathrm{R}_{2 \mathrm{k}} \\
\vdots & \vdots & \ddots & \vdots \\
0 & 0 & \cdots & \mathrm{R}_{\mathrm{kk}}
\end{array}\right)
$$

where, each $R_{\mathrm{ii}}$ is either a scalar or a $2 \times 2$ matrix. The scalar diagonal entries correspond to real eigenvalues and $2 \times 2$ matrices on the diagonal correspond to complex conjugate eigenvalues.

Remark: The matrix $\mathrm{R}$ in theorem 2 is known as the real Schur form of A.

Before we present the basic QR iteration method for computing the real Schur form of A we will present the QR factorization of A using Householder matrix.

Theorem 3 (Datta, 1995): Given an $n \times n$ matrix A, there exists an orthogonal matrix $\mathrm{Q}$ and an upper triangular matrix R such that:

$\mathrm{A}=\mathrm{QR}$

The matrix $\mathrm{Q}$ can be written as $\mathrm{Q}=\mathrm{H}_{1} \mathrm{H}_{2} \ldots \mathrm{H}_{\mathrm{n}-1}$ where each $\mathrm{H}_{\mathrm{i}}$ is a Householder matrix.

Definition 4 (Datta, 1995): A matrix of the form:

$$
\mathrm{H}=\mathrm{I}-\frac{2 \mathrm{uu}}{\mathrm{u}^{\mathrm{T}} \mathrm{u}}
$$

where, $\mathrm{u}$ is a nonzero vector, is called a Householder matrix.

Basic QR iteration (Datta, 1995): We first present the basic QR iteration method:

Set $\mathrm{A}_{0}=\mathrm{A}$ by:

Now compute a sequence of matrices $\left(A_{k}\right)$ defined

$\mathrm{A}_{0}=\mathrm{Q}_{0} \mathrm{R}_{0}$ (Using theorem 3)

$$
\mathrm{A}_{1}=\mathrm{R}_{0} \mathrm{Q}_{0}=\mathrm{Q}_{1} \mathrm{R}_{1}
$$$$
\mathrm{A}_{2}=\mathrm{R}_{1} \mathrm{Q}_{1}=\mathrm{Q}_{2} \mathrm{R}_{2}
$$

In general:

$$
\mathrm{A}_{\mathrm{K}}=\mathrm{Q}_{\mathrm{K}} \mathrm{R}_{\mathrm{K}}=\mathrm{R}_{\mathrm{K}-1} \mathrm{Q}_{\mathrm{K}-1} \mathrm{k}=1,2 \ldots
$$

The matrices in the sequence $\left\{A_{k}\right\}$ have a very interesting property. Each matrix in the sequence is orthogonally similar to the previous one and is, therefore, orthogonally similar to the original matrix. For example:

$$
\begin{aligned}
& \mathrm{A}_{1}=\mathrm{R}_{0} \mathrm{Q}_{0}=\mathrm{Q}_{0}{ }^{\mathrm{T}} \mathrm{A}_{0} \mathrm{Q}_{0} \text { (Because } \mathrm{Q}_{0}{ }^{\mathrm{T}} \mathrm{A}_{0}=\mathrm{R}_{0} \text { ) } \\
& \mathrm{A}_{2}=\mathrm{R}_{1} \mathrm{Q}_{1}=\mathrm{Q}_{1}{ }^{\mathrm{T}} \mathrm{A}_{1} \mathrm{Q}_{1}
\end{aligned}
$$

Thus, $A_{1}$ is orthogonally similar to $A$ and $A_{2}$ is orthogonally similar to $A_{1}$. Therefore, $A_{2}$ is orthogonally similar to $\mathrm{A}$, as the following computation shows:

$$
\mathrm{A}_{2}=\mathrm{Q}_{1}{ }^{\mathrm{T}} \mathrm{A}_{1} \mathrm{Q}_{1}=\mathrm{Q}_{1}{ }^{\mathrm{T}}\left(\mathrm{Q}_{0}{ }^{\mathrm{T}} \mathrm{A}_{0} \mathrm{Q}_{0}\right) \mathrm{Q}_{1}=\left(\mathrm{Q}_{0} \mathrm{Q}_{1}\right)^{\mathrm{T}} \mathrm{A}_{0}\left(\mathrm{Q}_{0} \mathrm{Q}_{1}\right)
$$

Because each matrix is orthogonally similar to the original matrix $A$ therefore it has the same eigenvalues as $A$, then the sequence $\left\{A_{K}\right\}$ converges to a triangular matrix.

Theorem 5: The set of $(n \times n)$ square matrices $M C_{n}$, where:

$$
M C_{n}=\left\{\left[\beta_{\mathrm{ij}}(\mathrm{z})_{\mathrm{t}}\right]_{\mathrm{n} \times \mathrm{n}}: \mathrm{i}, \mathrm{j} \in \mathbf{Z}^{+}, \beta_{\mathrm{ij}}(\mathrm{z})_{\mathrm{t}} \in \mathbf{R}\right\}
$$

can be transformed to the set of ( $\mathrm{n} \times \mathrm{n})$ upper triangular matrices $\mathrm{MC}_{\mathrm{n}}$ ", where:

$$
M C_{n} "=\left\{\begin{aligned}
& {\left[\beta_{i j}(z)_{t}\right]_{n \times n}: \beta_{i j}(z)_{t}=} 0 \forall 1 \leq j<i \leq n, \\
& i, j \in \mathbf{Z}^{+}, \beta_{i j}(z)_{t} \in \mathbf{R}
\end{aligned}\right\}
$$

\section{Proof: Let}

$$
\mathrm{MC}_{\mathrm{n}}=\left\{\left[\beta_{\mathrm{ij}}(\mathrm{z})_{\mathrm{t}}\right]_{\mathrm{n} \times \mathrm{n}}: \mathrm{i}, \mathrm{j} \in \mathbf{Z}^{+}, \beta_{\mathrm{ij}}(\mathrm{z})_{\mathrm{t}} \in \mathbf{R}\right\}
$$

By using theorem 3 and the basic QR iteration we obtain the form $\mathrm{MC}_{\mathrm{n}}$ " which is the real Schur form of $\mathrm{MC}_{\mathrm{n}}$. 
Semigroup of $\mathrm{MC}_{\mathrm{n}}{ }$ : We are going to show that the set of upper triangular matrices $\mathrm{MC}_{\mathrm{n}}$ " satisfies all the axioms of a semigroup given in (Whitelaw, 1978) under matrix multiplication. In other words, we are going to show that

- $\quad \mathrm{MC}_{\mathrm{n}}{ }^{\prime}=\left\{\begin{array}{r}{\left[\beta_{\mathrm{ij}}(\mathrm{z})_{\mathrm{t}}\right]_{\mathrm{n} \times \mathrm{n}}: \beta_{\mathrm{ij}}(\mathrm{z})_{\mathrm{t}}=0 \forall 1 \leq \mathrm{j}<\mathrm{i} \leq \mathrm{n},} \\ \mathrm{i}, \mathrm{j} \in \mathbf{Z}^{+}, \beta_{\mathrm{ij}}(\mathrm{z})_{\mathrm{t}} \in \mathbf{R}\end{array}\right\}$ is closed with respect to matrix multiplication

- Matrix multiplication on $\mathrm{MC}_{\mathrm{n}}$ " is associative

Theorem 6: The set of $(n \times n)$ upper triangular matrices $\mathrm{MC}_{\mathrm{n}}$ " is a semigroup under matrix multiplication.

Proof: Using similar proof as given in theorem 1, we arrive at the proof of our theorem.

\section{RESULTS}

In this study, we have shown that Magnetic Contour Plane of Fuzzy Topographic Topological Mapping (FTTM) which contains the EEG signals during epileptic seizure can be transformed into a semigroup of upper triangular matrices under matrix multiplication.

\section{DISCUSSION}

We have shown that the EEG signals during epileptic seizure can be viewed as a semigroup of upper triangular matrices under matrix multiplication. We demonstrated the possibility of transforming from one structure to the other, namely from topological to algebraic structure of EEG signals during seizure.

\section{CONCLUSION}

This research will enable us to proceed further in identifying characteristics of EEG signals during epileptic seizure.

\section{ACKNOWLEDGEMENT}

Praise be to Allah, the Almighty for given us the strength and courage to proceed with our entire life. Faisal would like to thank his family for all her support and encouragement and Hadhramout University of Science and Technology for granting the scholarship during his study. We would like also to thank Ministry of Higher Education, Malaysia for granting us FRGS Vot. 78315.

\section{REFERENCES}

Ahmad, T., R.A. Fairuz, F. Zakaria, and H. Isa, 2008a. Selection of a subset of EEG channels of epileptic patient during seizure using PCA. Proceeding of the 7th WSEAS International Conference on Signal Processing, Robotics and Automation, Advanced Topics on Signal Processing, Robotics and Automation, Feb. 20-22, University of Cambridge, UK., pp: 270-273.

Ahmad, T., R.S. Ahmad, W.E.Z.W.A. Rahman, L.L. Yun and F. Zakaria, 2008b. Fuzzy topographic topological mapping for localization simulated multiple current sources of MEG. J. Interdiscip. Math., $\quad$ 11: 381-393. http://www.connectjournals.com/jim

Datta, B.N., 1995. Numerical Linear Algebra and Applications. 1st Edn., Brooks/Cole Pub., ISBN: 13: 9780534174668, pp: 680.

Nehaniv, C. and K. Dautenhahn, 1998. Embodiment and memories-algebras of time and history for autobiographic agents. Proceeding of the 14th European Meeting on Cybernetics and Systems, Apr. 14-17, Austrian Society for Cybernetic Studies, Vienna, Austria, pp: 651-656.

Whitelaw, T.A., 1978. Introduction to Abstract Algebra. 2nd Edn., Blackie, Glasgow, ISBN: 0216904889, pp: 166.

Yun, L.L. and T. Ahmad, 2003. Construction of the sphere to elipsoid homeomorfisma. Math, 19: 121-138. http://www.fs.utm.my/matematika/images/stories/ matematika/200319205.pdf 